Original paper

\title{
New data for metakirchheimerite from Jáchymov (St. Joachimsthal), Czech Republic
}

\author{
Jakub PLÁŠIL 1*, Jiří ČEJKA², Jiří SEJKORA', Jan HLOUŠEK³, Viktor GOLIÁŠ4 \\ ${ }^{1}$ Department of Mineralogy and Petrology, National museum, Václavské nám. 68, 11579 Prague 1, Czech Republic; jakub_plasil@nm.cz \\ ${ }^{2}$ Natural History Museum, National Museum, Václavské náměstí 68, 11579 Prague 1, Czech Republic \\ ${ }^{3}$ U Roháčových kasáren 24, 10000 Prague 10, Czech Republic \\ ${ }^{4}$ Institute of Geochemistry, Mineralogy and Mineral Resources, Faculty of Science, Charles University in Prague, Albertov 6, 12843 \\ Prague 2, Czech Republic \\ * Corresponding author
}

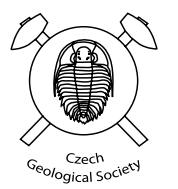

Metakirchheimerite was found only on a few samples from the Jan Evangelista vein at the "Adit level" of the Svornost shaft, Jáchymov (St. Joachimsthal) ore district, Krušné Hory/Erzgebirge Mountains, Czech Republic. It forms rare crystalline aggregates consisting of fine tabular crystals reaching $0.1 \mathrm{~mm}$ in size, which are very brittle, having light pink to light orange colour, with a high vitreous lustre. Metakirchheimerite crystalline aggregates occur on carbonate gangue with disseminated primary minerals: arsenopyrite, uraninite and skutterudite. Studied metakirchheimerite is a (sub-)recent alteration product of the primary minerals, connected to the old open mine adit environment. According to electron microprobe analyses, the empirical formula of metakirchheimerite can be expressed, based on $\mathrm{As}+\mathrm{P}=2$ apfu, as: $\left.\left(\mathrm{Co}_{0.53} \mathrm{Mg}_{0.25} \mathrm{Ni}_{0.08} \mathrm{Zn}_{0.07} \mathrm{Fe}_{0.05} \mathrm{Ca}_{0.03}\right)_{\Sigma 1.01}\left(\mathrm{UO}_{2}\right)_{2.07}\left[\left(\mathrm{AsO}_{4}\right)_{1.99}\left(\mathrm{PO}_{4}\right)_{0.01}\right)\right]_{\Sigma 2.00} .8 \mathrm{H}_{2} \mathrm{O}$. Metakirchheimerite from Jáchymov exhibits an extensive cationic substitution trend of the metanovácekite component. The X-ray diffraction pattern of metakirchheimerite is consistent with a triclinic symmetry inferred from the single-crystal study of the synthetic metakirchheimerite analogue. Refined unit-cell parameters for the triclinic space group $P-1$ are $a$ 7.210(4) $\AA, b$ 9.771(6) $\AA, c$ 13.252(9) $\AA, \alpha 75.39(4)^{\circ}, \beta 83.94(6)^{\circ}, \gamma 81.88(6)^{\circ}$ with unit-cell volume $V$ 892(1) $\AA^{3}$. Both infrared and Raman spectra of metakirchheimerite exhibited stretching and bending vibrations of $\left(\mathrm{UO}_{2}\right)^{2+},\left(\mathrm{AsO}_{4}\right)^{3-},\left(\mathrm{PO}_{4}\right)^{3-}$ and $\mathrm{H}_{2} \mathrm{O}$ groups. The vibration bands of all these units were tentatively assigned. The $\mathrm{U}-\mathrm{O}$ bond lengths in uranyl and $\mathrm{O}-\mathrm{H} \ldots \mathrm{O}$ hydrogen bond lengths, which were inferred from the spectra, are consistent with published crystal structure data for a synthetic analogue of metakirchheimerite.

Keywords: metakirchheimerite, X-ray powder data, chemical composition, vibration spectroscopy, uranyl bond lengths, hydrogen bond lengths

Received: 5 October 2009; accepted 18 December 2009; handling editor: M. Novák

The online version of this article (doi: 10.3190/jgeosci.057) contains supplementary electronic material.

\section{Introduction}

The importance of understanding uranium mineralogy and chemistry is closely connected with the broad issue of spent nuclear fuel ("SNF") alteration (Finch and Ewing 1992; Wronkiewicz et al. 1992, 1996; Finn et al. 1996; Murakami et al. 1997; Finch et al. 1999; Fayek et al. 2000; Shueneman et al. 2003; Procházka 2007; Procházka et al. 2009), waste management and environmental issues (e.g. Catalano et al. 2004, 2006). Hydrated uranyl arsenates of divalent transition metals are more or less abundant minerals of the secondary origin in the supergene zones of most uranium deposits (Finch and Murakami 1999). A group of hydrated uranyl arsenates and phosphates, with autunite-type sheet containing divalent cations in their interlayer, is represented by a general formula ${ }^{[6]} M^{2+}\left(\mathrm{UO}_{2}\right)_{2}\left(T \mathrm{O}_{4}\right)_{2} \cdot n \mathrm{H}_{2} \mathrm{O}(T$ is $\mathrm{P}$ or As), where $n$ is 12,10 and 8 most frequently. Divalent cations including
$\mathrm{Co}, \mathrm{Ni}, \mathrm{Mg}, \mathrm{Fe}, \mathrm{Mn}$ and $\mathrm{Zn}$ occupy six fold-coordinated $M^{2+}$ sites (Locock et al. 2004; Locock 2007a, b).

This paper presents new mineralogical data on a rare hydrated cobalt uranyl arsenate - metakirchheimerite, found recently in Jáchymov (St. Joachimsthal), Czech Republic. No recent and detailed data for metakirchheimerite have been published on natural specimens since Walenta's studies (Walenta 1958, 1964).

\section{Previous studies}

Metakirchheimerite was described as a new mineral by Walenta (1958) from the dump of the Sophia shaft in Wittichen, Baden, Germany. On the type locality metakirchheimerite forms coatings of tetragonal tabular crystals coming in association with metakahlerite, nováčekite, metaheinrichite and erythrite on weathered uraninite. 
These coatings have light pink colour, single crystals are green-yellow to colourless. Metakirchheimerite is non-pleochroic, with pearly lustre on cleavage planes; cleavage is excellent along (001). According to Walenta (1958), metakirchheimerite is optically uniaxial or biaxial, negative, with $\omega 1.644(2), \varepsilon 1.617(2)$ and $2 \mathrm{~V}$ of $0-20^{\circ}$. The chemical analyses proved $\mathrm{Co}, \mathrm{U}$ and $\mathrm{As}$ as the main constituents of metakirchheimerite, accompanied by minor Fe and traces of Ni. Walenta (1958) proposed the ideal formula $\mathrm{Co}(\mathrm{UO})_{2}\left(\mathrm{AsO}_{4}\right)_{2} \cdot 8 \mathrm{H}_{2} \mathrm{O}$, and assigned metakirchheimerite to metatorbernite group. The mineral is named to honour German geologist and palaeontologist Franz Waldemar Kirchheimer (1917-1984), an author of an important uranium monography (Kirchheimer 1963). Walenta (1964) published powder diffraction data for natural metakirchheimerite and for a synthetic dodecahydrate phase "kirchheimerite" - not approved as (a valid) mineral. Weight loss obtained for metakirchheimerite from thermal analysis corresponds to the sum of 15 wt. \%, and the content of 8.59 moles $\mathrm{H}_{2} \mathrm{O}$ in its crystal structure. The DTA curve shows endotherms at 115 , 195,300 and $1005^{\circ} \mathrm{C}$, and slight exotherms at $>620^{\circ} \mathrm{C}$. Regarding the powder X-ray diffraction, Walenta (1964) mentioned problem with indexing the powder pattern. He stated that this problem could be solved after doubling the $c$ dimension of the unit-cell and correction of the $a$ parameter. Since Walenta's times, further scientific papers were based on the study of synthetic metakirchheimerite analogues. Nabar and Iyer (1977) reported preparation of octahydrates of zinc, nickel and cobalt uranylarsenates and their physico-chemical properties. Based on systematic absences in powder diffraction patterns, they assigned synthetic metakirchheimerite to the space group $P 4_{2} / m$, with unit-cell parameters $a 6.98(2) \AA$ and $c$ 16.93(2) $\AA$, the unit-cell volume $V 825 \AA^{3}$ and density of $4.042 \mathrm{~g} \cdot \mathrm{cm}^{-3}$ (measured) and $4.111 \mathrm{~g} \cdot \mathrm{cm}^{-3}$ (calculated). Vochten and Goeminne (1984) published the synthesis, crystallographic data, solubility products and electrokinetic properties of synthetic metazeunerite, metakirchheimerite and, by that time unknown in natural occurrence, uranylarsenate of nickel (corresponding to new mineral IMA-2008-50 - metarauchite, Plášil et al. 2008; Plášil 2009). Vochten and Goeminne (1984) described synthetic metakirchheimerite as heptahydrate, which is in contrary both to the results of Walenta (1964) and to the currently used definition. Based on X-ray powder diffraction data, they reported that assignment of $h k l$ indices is not reliable, if it is based of unit-cell parameters given by Walenta (1964). Moreover, none of the three published powder diffraction patterns is consistent, differing namely in number of diffractions and intensities (Walenta 1964; Vochten and Goeminne 1984; Nabar and Iyer 1977). Based on the results of thermal analysis, Vochten and Goeminne (1984) described thermal dehydration of the synthetic metakirchheimerite in a following way: phase containing $7 \mathrm{H}_{2} \mathrm{O} \rightarrow\left(45^{\circ} \mathrm{C}\right)$ phase with $6 \mathrm{H}_{2} \mathrm{O} \rightarrow(122$ $\left.{ }^{\circ} \mathrm{C}\right)$ phase with $2.5 \mathrm{H}_{2} \mathrm{O} \rightarrow\left(275{ }^{\circ} \mathrm{C}\right)$ anhydrous phase. The authors did not assign the vibration bands for the spectra of the individual phases and confined themselves to the statement that vibrational properties of hydrated $\mathrm{Ni}$ and Co uranyl arsenates are practically identical (Vochten and Goeminne 1984). Ondruš et al. (2003c) published a short notice about metakirchheimerite from the Jan Evangelista vein in the Jáchymov ore district, without any further details.

Locock et al. (2004) reported solved crystal structure for a synthetic analogue of metakirchheimerite (named as "CoUAs8") and also for synthetic dodecahydrate, corresponding to up to now species with doubtful status, "kirchheimerite". In the asymmetric part of the synthetic metakirchheimerite unit-cell, two structurally non-equivalents $\mathrm{U}$ atoms, two non-equivalent As atoms, a single $\mathrm{Co}$ atom, and $20 \mathrm{O}$ atoms are present. The crystal structure (Fig. 1) consists of the uranyl tetragonal bipyramids $\left(\mathrm{UO}_{2}\right) \mathrm{O}_{4}$ linked by arsenate tetrahedra along $(011)$, resulting in sheets of the autunite topology. Cobalt-based octahedra in the interlayer are formed by five $\mathrm{H}_{2} \mathrm{O}$ groups and one shared uranyl-apical oxygen atom that links the octahedron directly to the structural sheets. In addition, three non-equivalent $\mathrm{H}_{2} \mathrm{O}$ molecules are located in the interlayer, held only by hydrogen bonding.

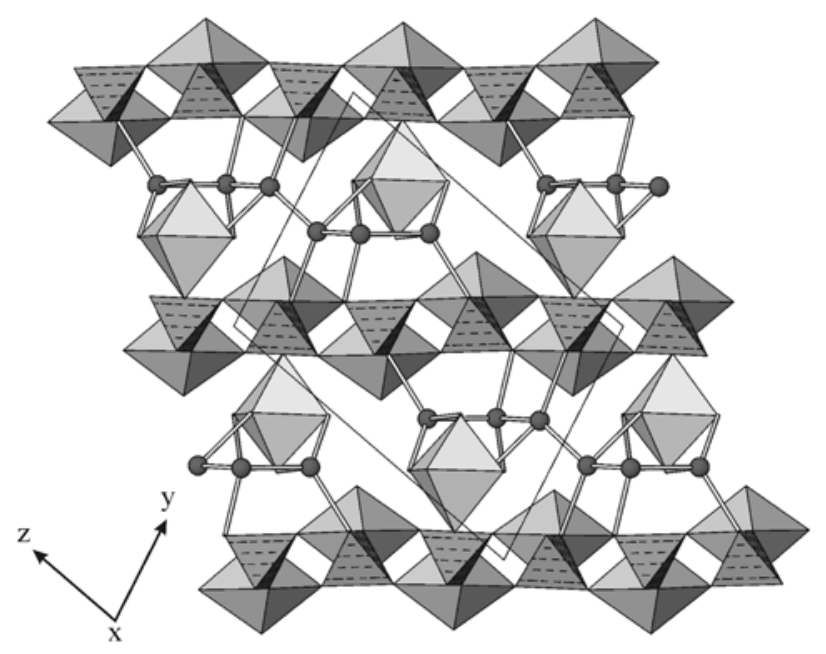

Fig. 1 The crystal structure of synthetic metakirchheimerite "CoUAs8" (Locock et al. 2004) in projection along [100]. The uranyl arsenate sheets are parallel to (011). Uranyl tetragonal bipyramids are grey, arsenate tetrahedra are dark grey and stippled, cobalt-based octahedra are light-grey and groups of molecular water are dark grey spheres. Donor-acceptor (O...O) distances $(<3.0 \AA)$ corresponding to probable hydrogen bonds are shown as rods (Locock et al. 2004, the picture courtesy of Andrew Locock). 


\section{Occurrence}

Samples containing the later identified metakirchheimerite originate from the Jan Evangelista (Johannes Evangelist) vein at the "Adit level" of the Svornost shaft, the Jáchymov ore district, Krušné Hory Mts., Bohemia, Czech Republic. The Jan Evangelista vein is one of the most important in the Svornost mine-area, carrying both $\mathrm{Ag}-\mathrm{As}-$ $\mathrm{Bi}-\mathrm{Co}-\mathrm{Ni}$ and $\mathrm{U}$ mineralization, which were intensively mined since $16^{\text {th }}$ till $20^{\text {th }}$ century. Detailed geological settings and characteristics of the veins and mineralization were described mainly by Ondruš et al. (2003a-d).

Two samples, represented only by fragments approximately $1 \times 1 \times 1.5 \mathrm{~cm}$ in size, consist of the carbonate gangue (dominantly calcite and minor dolomite) with disseminated small grains of primary mineralization, represented mainly by skutterudite, uraninite and arsenopyrite. The surfaces of these fragments are coated by small crystals and crystal aggregates of metakirchheimerite

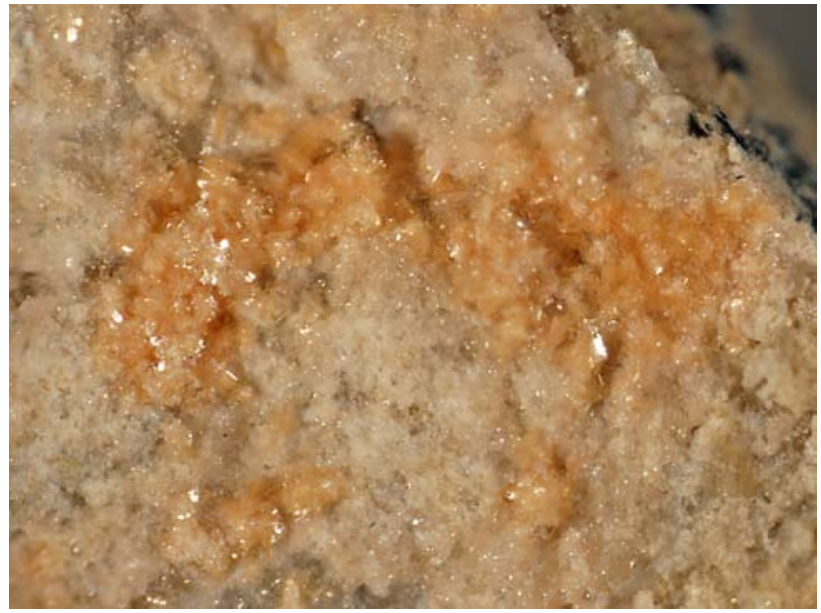

Fig. 2 Aggregates of metakirchheimerite on a surface of altered gangue from Jáchymov. Width of photograph is $4 \mathrm{~mm}$. Photo by J. Sejkora (Nikon SMZ 1500).

(Fig. 2). The aggregates are up to $2 \mathrm{~mm}$ long, consisting of fine tabular crystals, maximally up to $0.1 \mathrm{~mm}$ (Fig. 3). The crystals are of light pink to grey-pink colour, with a pearly lustre. Crystals are very brittle with excellent cleavage, corresponding with pinacoid (011) (Fig. 4).

\section{Results}

\subsection{X-ray powder diffraction}

A hand-picked sample of metakirchheimerite was prepared for the X-ray powder diffraction experiment using the continuous mode of the PANalytical X'Pert Pro

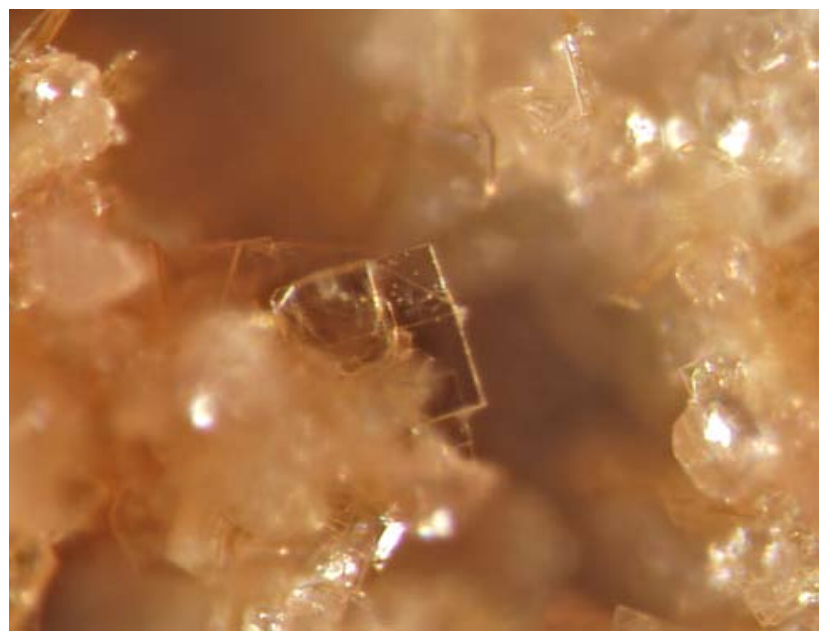

Fig. 3 Detail of thin tabular, translucent crystal of metakirchheimerite. Width of photograph is $0.1 \mathrm{~mm}$. Photo by J. Sejkora (Nikon SMZ $1500)$.

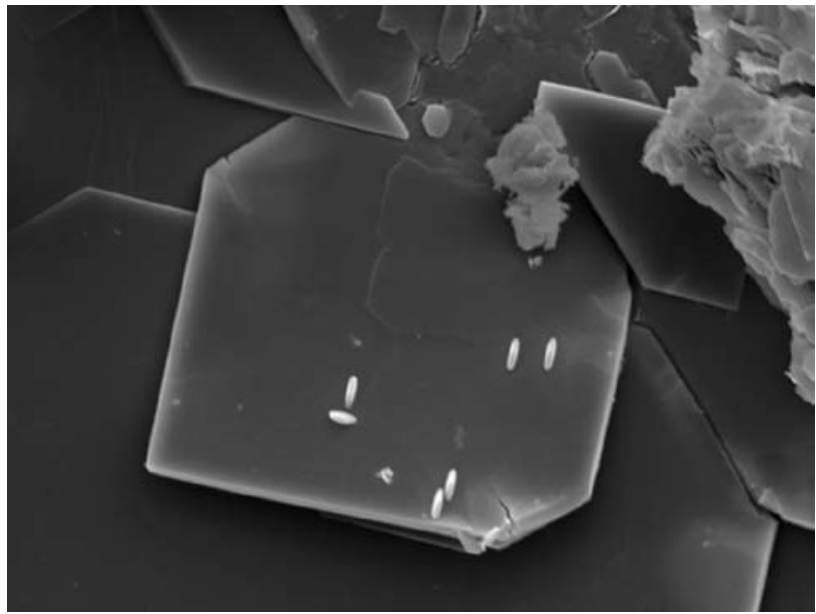

Fig. 4 Detail of metakirchheimerite crystal morphology showing dominating face (011). Other crystal faces suggest symmetry to be lower than tetragonal. Small aggregates on the surface of the crystal are probably scorodite crystals (according to EDS). Width of secondary electron (SE) image is $40 \mu \mathrm{m}$. Photo by J. Plášil (JEOL JSM 6380).

powder diffractometer operating at $40 \mathrm{kV}$ and $30 \mathrm{~mA}$, equipped with the $\mathrm{X}^{\prime}$ Celerator detector and the secondary graphite monochromator. The powder diffraction pattern obtained with the Bragg-Bretano geometry exhibited effects of a strong preferred orientation due to the excellent (011) cleavage of metakirchheimerite. This effect was particularly notable as high intensities of the diffraction maxima of the type $0 \mathrm{kl}$, whereas profiles of other diffractions had a low intensity and were poorly defined. To avoid these problems, experiment with the DebyeScherrer geometry was undertaken. The effects of the preferred orientation were nearly eliminated, but the chart is characterised by a lower definition of diffraction profiles, 
Tab. 1 Powder diffraction patterns for metakirchheimerite from Jáchymov

\begin{tabular}{|c|c|c|c|c|c|c|c|c|c|c|c|c|}
\hline \multicolumn{5}{|c|}{ Calculated from the crystal structure of "CoUAs8"* } & \multicolumn{3}{|c|}{ Jáchymov $^{1}$} & \multicolumn{3}{|c|}{ Jáchymov $^{2}$} & \multicolumn{2}{|c|}{ Walenta (1964) } \\
\hline$I_{\text {calc }}$ & $h$ & $\boldsymbol{k}$ & $l$ & $\boldsymbol{d}_{\text {calc }}$ & $I_{o b s}$ & $d_{o b s}$ & $d_{\text {calc }}$ & $I_{\text {obs }}$ & $d_{o b s}$ & $d_{\text {calc }}$ & $I_{\text {obs }}$ & $d_{o b s}$ \\
\hline 2 & 0 & 1 & 0 & 9.39 & & & & & & & & \\
\hline 100 & 0 & 1 & 1 & 8.61 & 100 & 8.66 & 8.62 & 100 & 8.61 & 8.61 & 10 & 8.75 \\
\hline 1 & 0 & -1 & 1 & 6.83 & & & & & & & & \\
\hline 1 & 1 & 0 & 1 & 6.40 & & & & & & & & \\
\hline 2 & 0 & 0 & 2 & 6.39 & & & & & & & & \\
\hline 1 & -1 & 0 & 1 & 6.03 & & & & & & & & \\
\hline 1 & 0 & 1 & 2 & 5.99 & & & & & & & & \\
\hline 5 & 1 & 1 & 1 & 5.97 & & & & & & & & \\
\hline 1 & 1 & -1 & 0 & 5.35 & & & & & & & & \\
\hline 12 & -1 & 1 & 1 & 5.09 & & & & & & & & \\
\hline 16 & -1 & -1 & 1 & 5.06 & 12 & 5.07 & 5.05 & & & & 6 & 5.08 \\
\hline 12 & 1 & 0 & 2 & 4.93 & 9 & 4.95 & 4.94 & & & & & \\
\hline 8 & 1 & -1 & 1 & 4.80 & & & & & & & & \\
\hline 17 & 0 & 2 & 0 & 4.69 & 9 & 4.76 & 4.70 & & & & 3 & 4.69 \\
\hline 4 & -1 & 0 & 2 & 4.59 & & & & & & & & \\
\hline 8 & 0 & 2 & 2 & 4.31 & 15 & 4.31 & 4.31 & 8 & 4.31 & 4.31 & 6 & 4.32 \\
\hline 18 & 0 & 1 & 3 & 4.28 & 17 & 4.29 & 4.29 & 2 & 4.28 & 4.28 & & \\
\hline 18 & 1 & 2 & 2 & 3.98 & 11 & 3.97 & 3.98 & $<1$ & 3.99 & 3.98 & 2 & 3.99 \\
\hline 1 & 1 & -1 & 2 & 3.95 & & & & & & & & \\
\hline 26 & 0 & -1 & 3 & 3.57 & 23 & 3.57 & 3.57 & $<1$ & 3.57 & 3.57 & 9 & 3.58 \\
\hline 24 & 2 & 0 & 0 & 3.55 & & & & & & & & \\
\hline 20 & 2 & 1 & 1 & 3.49 & 14 & 3.49 & 3.49 & & & & & \\
\hline 13 & -1 & 2 & 2 & 3.44 & 10 & 3.46 & 3.45 & $<1$ & 3.44 & 3.44 & 6 & 3.44 \\
\hline 16 & 0 & -2 & 2 & 3.41 & 11 & 3.42 & 3.41 & $<1$ & 3.42 & 3.42 & & \\
\hline 9 & 0 & 3 & 1 & 3.22 & & & & $<1$ & 3.23 & 3.23 & 2 & 3.23 \\
\hline 10 & 0 & 0 & 4 & 3.20 & 7 & 3.19 & 3.20 & & & & & \\
\hline 7 & -2 & 1 & 1 & 3.11 & 6 & 3.12 & 3.12 & & & & $<1$ & 3.12 \\
\hline 3 & 2 & 2 & 0 & 3.02 & & & & & & & & \\
\hline 4 & 1 & -2 & 2 & 3.02 & 11 & 3.02 & 3.02 & & & & 5 & 3.01 \\
\hline 3 & -2 & 0 & 2 & 3.02 & & & & & & & & \\
\hline 6 & 0 & 2 & 4 & 2.995 & 3 & 2.997 & 2.999 & $<1$ & 2.993 & 2.994 & & \\
\hline 2 & 1 & 0 & 4 & 2.994 & & & & & & & & \\
\hline 5 & 2 & 2 & 2 & 2.985 & & & & & & & & \\
\hline 2 & 2 & 1 & 3 & 2.922 & & & & & & & & \\
\hline 3 & 0 & -3 & 1 & 2.885 & & & & & & & & \\
\hline 1 & -2 & -1 & 2 & 2.864 & & & & & & & & \\
\hline 2 & -1 & 0 & 4 & 2.840 & & & & & & & & \\
\hline 4 & 1 & 3 & 3 & 2.824 & & & & $<1$ & 2.824 & 2.827 & & \\
\hline 3 & 1 & 3 & -1 & 2.769 & & & & & & & & \\
\hline 3 & 2 & -2 & 0 & 2.677 & $<1$ & 2.684 & 2.685 & & & & & \\
\hline 1 & 0 & 1 & 5 & 2.631 & & & & & & & & \\
\hline 1 & -1 & 2 & 4 & 2.627 & & & & & & & & \\
\hline 2 & 1 & -3 & 1 & 2.586 & 5 & 2.583 & 2.590 & $<1$ & 2.583 & 2.586 & & \\
\hline 2 & 2 & 3 & 1 & 2.580 & & & & & & & & \\
\hline 2 & -2 & 1 & 3 & 2.577 & & & & $<1$ & 2.576 & 2.577 & & \\
\hline 2 & 1 & 1 & 5 & 2.558 & & & & & & & & \\
\hline 1 & -2 & 2 & 2 & 2.546 & & & & & & & & \\
\hline 8 & 2 & -1 & 3 & 2.533 & 8 & 2.539 & 2.541 & $<1$ & 2.534 & 2.532 & 5 & 2.53 \\
\hline 7 & -2 & -2 & 2 & 2.528 & 5 & 2.518 & 2.524 & & & & & \\
\hline 2 & -1 & 3 & 3 & 2.524 & & & & & & & & \\
\hline 6 & -2 & -1 & 3 & 2.503 & & & & & & & & \\
\hline 2 & 2 & 2 & 4 & 2.473 & & & & & & & & \\
\hline 5 & 2 & 0 & 4 & 2.463 & 3 & 2.474 & 2.470 & & & & & \\
\hline 2 & 2 & -2 & 2 & 2.399 & & & & & & & & \\
\hline 2 & -1 & 1 & 5 & 2.385 & 3 & 2.384 & 2.386 & & & & & \\
\hline
\end{tabular}




\begin{tabular}{|c|c|c|c|c|c|c|c|c|c|c|c|c|}
\hline \multicolumn{5}{|c|}{ Calculated from the crystal structure of "CoUAs8"* } & \multicolumn{3}{|c|}{ Jáchymov $^{1}$} & \multicolumn{3}{|c|}{ Jáchymov $^{2}$} & \multicolumn{2}{|c|}{ Walenta (1964) } \\
\hline$I_{\text {calc }}$ & $h$ & $k$ & $l$ & $\boldsymbol{d}_{\text {calc }}$ & $I_{\text {obs }}$ & $d_{o b s}$ & $d_{\text {calc }}$ & $I_{\text {obs }}$ & $d_{o b s}$ & $d_{\text {calc }}$ & $I_{o b s}$ & $d_{o b s}$ \\
\hline 2 & -2 & -3 & 1 & 2.355 & & & & & & & & \\
\hline 2 & 0 & -3 & 3 & 2.276 & & & & & & & & \\
\hline 3 & -3 & -1 & 1 & 2.274 & 7 & 2.275 & 2.274 & & & & 5 & 2.27 \\
\hline 4 & 3 & 0 & 2 & 2.273 & & & & & & & & \\
\hline 4 & -1 & -2 & 4 & 2.272 & & & & & & & & \\
\hline 2 & 1 & -2 & 4 & 2.258 & & & & & & & & \\
\hline 2 & 1 & 3 & 5 & 2.256 & & & & & & & & \\
\hline 2 & 1 & -1 & 5 & 2.236 & & & & & & & & \\
\hline 3 & 3 & 2 & 2 & 2.231 & 2 & 2.235 & 2.234 & & & & & \\
\hline 2 & 3 & 2 & 0 & 2.227 & & & & & & & & \\
\hline 3 & -3 & 1 & 1 & 2.194 & 1 & 2.199 & 2.198 & & & & & \\
\hline 3 & -1 & 4 & 2 & 2.172 & & & & & & & & \\
\hline 1 & 1 & 4 & 4 & 2.159 & 2 & 2.163 & 2.161 & & & & & \\
\hline 4 & 0 & 4 & 4 & 2.153 & 10 & 2.152 & 2.155 & 2 & 2.155 & 2.154 & 6 & 2.15 \\
\hline 2 & 1 & -4 & 0 & 2.151 & & & & & & & & \\
\hline 2 & 2 & 4 & 2 & 2.144 & 1 & 2.143 & 2.143 & 1 & 2.148 & 2.148 & & \\
\hline 2 & -2 & 2 & 4 & 2.142 & & & & & & & & \\
\hline 3 & 0 & 0 & 6 & 2.130 & & & & & & & & \\
\hline 2 & 1 & 2 & 6 & 2.127 & & & & & & & & \\
\hline 3 & -1 & 3 & 5 & 2.066 & & & & $<1$ & 2.067 & 2.066 & 1 & 2.05 \\
\hline 3 & 0 & -4 & 2 & 2.051 & & & & & & & & \\
\hline 2 & 3 & 3 & 3 & 1.9901 & & & & & & & & \\
\hline 1 & -1 & 4 & 4 & 1.9738 & & & & $<1$ & 1.9732 & 1.9736 & & \\
\hline 2 & -3 & 2 & 2 & 1.9464 & & & & & & & & \\
\hline 3 & 1 & 5 & 3 & 1.9046 & $<1$ & 1.9073 & 1.9053 & $<1$ & 1.9075 & 1.9075 & & \\
\hline 2 & 0 & 1 & 7 & 1.8781 & & & & & & & & \\
\hline 2 & 3 & 1 & 5 & 1.8615 & & & & & & & & \\
\hline 2 & -2 & 4 & 2 & 1.8453 & & & & & & & & \\
\hline 2 & 1 & 4 & 6 & 1.8193 & & & & $<1$ & 1.8190 & 1.8203 & & \\
\hline 4 & 4 & 1 & 1 & 1.7970 & 4 & 1.7990 & 1.7996 & & & & & \\
\hline 3 & 0 & -2 & 6 & 1.7859 & 2 & 1.7852 & 1.7859 & & & & 4 & 1.787 \\
\hline 3 & 0 & -3 & 5 & 1.7822 & & & & & & & & \\
\hline 2 & 4 & 0 & 0 & 1.7754 & & & & & & & & \\
\hline 2 & -2 & 0 & 6 & 1.7724 & & & & & & & & \\
\hline 1 & 0 & 3 & 7 & 1.7718 & 1 & 1.7748 & 1.7743 & $<1$ & 1.7617 & 1.7624 & & \\
\hline 2 & -1 & 5 & 3 & 1.7621 & & & & & & & & \\
\hline 1 & 2 & 1 & 7 & 1.7311 & & & & & & & $<1$ & 1.726 \\
\hline 2 & -1 & 4 & 6 & 1.6894 & 2 & 1.6904 & 1.6918 & $<1$ & 1.6896 & 1.6890 & 2 & 1.687 \\
\hline 1 & 4 & 3 & 1 & 1.6602 & $<1$ & 1.6596 & 1.6602 & & & & & \\
\hline 1 & 1 & -4 & 4 & 1.6404 & $<1$ & 1.6412 & 1.6419 & & & & & \\
\hline 2 & -4 & -2 & 2 & 1.6101 & 3 & 1.6089 & 1.6090 & & & & & \\
\hline 2 & -2 & -3 & 5 & 1.6067 & 4 & 1.6047 & 1.6039 & & & & 4 & 1.602 \\
\hline 2 & 2 & -2 & 6 & 1.6031 & & & & & & & & \\
\hline 2 & 4 & 0 & 4 & 1.6005 & & & & & & & & \\
\hline 2 & 4 & -1 & 3 & 1.5975 & & & & & & & & \\
\hline 1 & 1 & 6 & 4 & 1.5767 & 2 & 1.5771 & 1.5776 & & & & 2 & 1.565 \\
\hline 2 & 1 & 6 & 0 & 1.5695 & & & & & & & & \\
\hline 2 & -3 & 4 & 2 & 1.5686 & & & & & & & & \\
\hline 2 & 3 & 4 & 6 & 1.5571 & & & & & & & & \\
\hline 1 & 1 & 5 & 7 & 1.5166 & $<1$ & 1.4915 & 1.4915 & & & & $<1$ & 1.516 \\
\hline 1 & -1 & 3 & 9 & 1.3665 & 2 & 1.3687 & 1.3679 & & & & & \\
\hline
\end{tabular}

Debye-Scherrer, $5-100^{\circ} 2 \theta \mathrm{Cu} K_{\alpha}$ radiation, integrated step $0.02^{\circ} / 2500 \mathrm{~s}$, rotated sample with a frequency of $1 \mathrm{~s}^{-1}$

Bragg-Brentano, $5-60^{\circ} 2 \theta \mathrm{Cu} K_{\alpha}$, integrated step $0.02^{\circ} / 2500 \mathrm{~s}$, rotated sample with a frequency of $2 \mathrm{~s}^{-1}$

Walenta (1964) - Debye-Scherrer pattern by Walenta (1964)

* calculated from the structural data of synthetic metakirchheimerite (Locock et al. 2004) Listed are diffractions either observed or with relative intensities higher or equal 2. 
a higher background, and a relatively low peak to background ratio. The position of each diffraction maximum in both diffraction patterns was refined using PearsonVII profile shape function by the Xfit software (Coelho and Cheary 1997). The unit-cell parameters were refined with the Celref software (Laugier and Bochu 2002).

The powder diffraction data obtained in the BraggBrentano and Debye-Scherrer settings are presented in Tab. 1 together with calculated theoretical values $d_{\text {calc }}$ obtained from the crystal structure data for synthetic metakirchheimerite (Locock et al. 2004). In addition, the diffraction data for natural metakirchheimerite published by Walenta (1964) are included in Tab. 1. Compared to the latter, the new diffraction data obtained in the current study are more complete. The refinement of the unit-cell parameters was based on the positions of 39 (DebyeScherrer) and 21 (Bragg-Brentano) diffraction maxima (Tab. 1) obtained from the profile fitting. $\mathrm{Hkl}$ indices were assigned to the observed diffractions on the basis of the theoretical positions, inferred from the crystal structure of synthetic metakirchheimerite (Locock et al. 2004). Both the sets of refined unit-cell parameters (Tab. 2) are approximately the same, varying only in the range of their sigma values, and are consistent with parameters given by Locock et al. (2004) for the synthetic analogue of metakirchheimerite.

Tab. 2 Refined unit-cell parameters for metakirchheimerite from Jáchymov compared with parameters for a synthetic analogue (for triclinic space group $P$-1)

\begin{tabular}{|c|c|c|c|}
\hline & \multicolumn{2}{|c|}{ Jáchymov, this work } & \multirow[t]{2}{*}{ Synt., Locock et al. (2004) } \\
\hline & 1 & 2 & \\
\hline$a[\AA]$ & $7.207(6)$ & $7.199(6)$ & $7.1955(3)$ \\
\hline$b[\AA]$ & $9.773(6)$ & $9.786(1)$ & $9.7715(4)$ \\
\hline$c[\AA]$ & $13.25(1)$ & $13.23(1)$ & $13.2319(6)$ \\
\hline$\alpha\left[^{\circ}\right]$ & $75.43(6)$ & $75.55(4)$ & $75.525(1)$ \\
\hline$\beta\left[^{\circ}\right]$ & $83.89(7)$ & $84.05(7)$ & $84.052(1)$ \\
\hline$\gamma\left[{ }^{\circ}\right]$ & $81.83(8)$ & $81.51(6)$ & 81.661(1) \\
\hline$V\left[\AA^{3}\right]$ & $892(1)$ & $890(1)$ & $889.08(7)$ \\
\hline
\end{tabular}

1 - Debye-Scherrer, mean-squared deviation on refined positions $0.06^{\circ} 2 \theta$

2 - Bragg-Brentano, mean-squared deviation on refined positions $0.02{ }^{\circ} 2 \theta$

\subsection{Chemical composition}

Chemical composition of metakirchheimerite was studied using an electron microprobe Cameca SX100 (Laboratory of Electron Microscopy and Microanalysis of the Masaryk University and Czech Geological Survey, Brno, operator R. Škoda). Wavelength dispersive mode and operating voltage of $15 \mathrm{kV}, 2 \mathrm{nA}$ current and $20 \mu \mathrm{m}$ beam diameter were used. The following analytic lines and crystals were selected to minimize line overlap: $K_{\alpha}$ lines: $\mathrm{P}$ (fluorapatite), $\mathrm{Fe}$ (andradite), $\mathrm{Mg}\left(\mathrm{MgAl}_{2} \mathrm{O}_{4}\right), \mathrm{Zn}$ $(\mathrm{ZnO}), \mathrm{Ni}(\mathrm{Ni}), \mathrm{Co}(\mathrm{Co}) ; L_{\alpha}$ lines: As $(\mathrm{InAs}) ; M_{\beta}$ lines: $\mathrm{U}$ (U metallic). Peak counting times (CT) were 10-20 seconds for major elements, 40-60 seconds for minor to trace elements and counting time on background was $1 / 2$ CT. The measured intensities were converted to element concentrations using the PAP program (Pouchou and Pichoir 1985).

Chemical composition of metakirchheimerite from Jáchymov (Tab. 3) can be expressed by an empirical formula (mean of 3 analyses, $\mathrm{As}+\mathrm{P}=2$ apfu): $\left(\mathrm{Co}_{0.53} \mathrm{Mg}_{0.25} \mathrm{Ni}_{0.08}\right.$ $\left.\left.\mathrm{Zn}_{0.07} \mathrm{Fe}_{0.05} \mathrm{Ca}_{0.03}\right)_{\Sigma 1.01}\left(\mathrm{UO}_{2}\right)_{2.07}\left[\left(\mathrm{AsO}_{4}\right)_{1.99}\left(\mathrm{PO}_{4}\right)_{0.01}\right)\right]_{\Sigma 2.00}$ $.8 \mathrm{H}_{2} \mathrm{O}$.

Tab. 3 Chemical composition of metakirchheimerite from Jáchymov

\begin{tabular}{|c|c|c|c|c|}
\hline & Mean & 1 & 2 & 3 \\
\hline $\mathrm{CaO}$ & 0.16 & 0.14 & 0.22 & 0.13 \\
\hline $\mathrm{MgO}$ & 0.93 & 0.80 & 0.87 & 1.13 \\
\hline $\mathrm{FeO}$ & 0.33 & 0.29 & 0.56 & 0.14 \\
\hline $\mathrm{CoO}$ & 3.76 & 3.49 & 3.22 & 4.58 \\
\hline $\mathrm{NiO}$ & 0.55 & 0.52 & 0.63 & 0.50 \\
\hline $\mathrm{ZnO}$ & 0.55 & 0.72 & 0.36 & 0.57 \\
\hline $\mathrm{P}_{2} \mathrm{O}_{5}$ & 0.07 & 0.03 & 0.03 & 0.15 \\
\hline $\mathrm{As}_{2} \mathrm{O}_{5}$ & 21.54 & 21.08 & 21.51 & 22.04 \\
\hline $\mathrm{UO}_{3}$ & 55.42 & 58.86 & 55.15 & 52.25 \\
\hline $\mathrm{H}_{2} \mathrm{O}^{*}$ & 14.01 & - & - & - \\
\hline Total & 97.32 & 85.92 & 82.55 & 81.48 \\
\hline $\mathrm{Ca}$ & 0.031 & & & \\
\hline $\mathrm{Mg}$ & 0.245 & & & \\
\hline $\mathrm{Fe}$ & 0.049 & & & \\
\hline Co & 0.533 & & & \\
\hline $\mathrm{Ni}$ & 0.078 & & & \\
\hline $\mathrm{Zn}$ & 0.071 & & & \\
\hline$\Sigma A$ site & 1.001 & & & \\
\hline $\mathrm{P}$ & 0.011 & & & \\
\hline As & 1.989 & & & \\
\hline$\Sigma T$ site & 2.000 & & & \\
\hline $\mathrm{U}$ & 2.056 & & & \\
\hline $\mathrm{H}_{2} \mathrm{O}$ & 8.000 & & & \\
\hline
\end{tabular}

$\mathrm{H}_{2} \mathrm{O}^{*}$ - content of molecular water in wt. \% derived from theoretical $8 \mathrm{~mol} . \mathrm{H}_{2} \mathrm{O}$ in the crystal structure of metakirchheimerite Mean - based on mean of 3 analyses

Besides slightly dominant Co atoms $(0.49-0.63 \mathrm{Co}$ $a p f u)$ at $A$ site, the presence of several isomorphic components was observed (Fig. 5). These are represented namely by $\mathrm{Mg}$ (up to 0.29 apfu), $\mathrm{Ni}$ (up to $0.09 a p f u$ ) and $\mathrm{Zn}(0.10 \mathrm{apfu})$ contents, corresponding to metanováčkeite $(\mathrm{Mg})$, metarauchite $(\mathrm{Ni})$ and metalodèvite $(\mathrm{Zn})$ isomorphic components. Only negligible contents (up to 0.02 apfu) of $\left(\mathrm{PO}_{4}\right)^{3-}$ anions were detected, indicating major prevalence of $\left(\mathrm{AsO}_{4}\right)^{3-}$ ions in source fluids. The molecular water content could not have been assessed directly in 


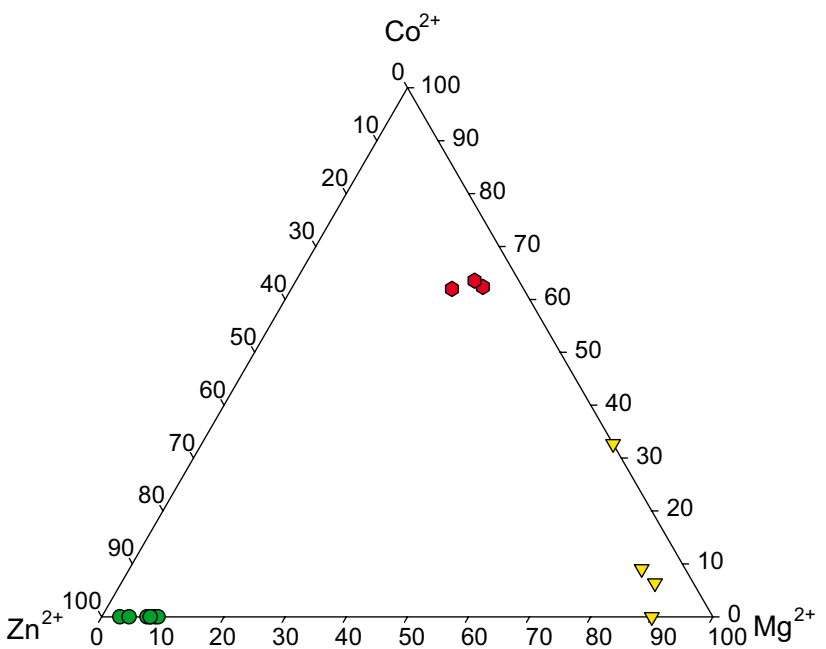

- metakirchheimerite, Jáchymov (this paper)

- metalodèvite, Príibram (Plášil 2009)

$\nabla \quad$ metanováčekite, Jáchymov (Plášil 2009)

Fig. 5 A ternary plot of the $A$-site occupancy (atomic ratio) for studied metakirchheimerite and related minerals.

the studied material due to small amount of the sample available. Therefore, the water content was inferred based on theoretical value for the synthetic metakirchheimerite, an octahydrate (Locock et al. 2004).

\subsection{Vibrational spectroscopy}

The infrared spectrum of metakirchheimerite was recorded by DRIFTS method on the Nicolet Magna 760 FTIR spectrometer (range 4000-600 $\mathrm{cm}^{-1}$, resolution $4 \mathrm{~cm}^{-1}$, 128 scans, Happ-Genzel apodization), equipped with Spectra Tech InspectIR micro FTIR accessory. Samples were mixed with $\mathrm{KBr}$ without using a pressure, to avoid dehydration or reactions in solid state, and immediately measured. The same $\mathrm{KBr}$ was measured as a reference. The Raman spectrum $\left(1100-170 \mathrm{~cm}^{-1}\right)$ was collected with a dispersive Raman spectrometer Labram HR (Jobin Yvon) with a confocal Olympus microscope. The Raman signal was excited by a $730 \mathrm{~nm}$ laser and detected with a multichannel air-cooled CCD camera. The laser power was limited to $10 \mathrm{~mW}$. Data processing was performed under Omnic Spectral tools software.

Neither infrared, nor Raman spectra of natural metakirchheimerite have been published yet. Vochten and Goeminne (1984) only mentioned that synthetic uranyl arsenates containing $\mathrm{Co}, \mathrm{Ni}$ and $\mathrm{Cu}$ exhibit the same vibrational characteristics. Chernorukov et al. (2000) published infrared spectra for synthetic metakirchheimerite and not approved "kirchheimerite" with assigned vibration bands. In the study of Plášil (2009), the infrared and
Tab. 4 Tentative assignment of metakirchheimerite infrared and Raman spectra

\begin{tabular}{|c|c|c|}
\hline Assignment & Infrared $\left[\mathrm{cm}^{-1}\right]$ & $\operatorname{Raman}\left[\mathrm{cm}^{-1}\right]$ \\
\hline \multirow{2}{*}{$v \mathrm{O}-\mathrm{H}$ stretching } & 3521 (s-sharp) & - \\
\hline & 3123 (s-broad) & - \\
\hline \multirow{2}{*}{$v_{2}(\delta)$ bending $\mathrm{H}-\mathrm{O}-\mathrm{H}$} & $1622(\mathrm{w})$ & - \\
\hline & $1586(w)$ & - \\
\hline \multirow{3}{*}{$v_{3}\left(\mathrm{PO}_{4}\right)^{3-}$} & 1109 (vw) & - \\
\hline & $1065(w)$ & - \\
\hline & $1007(\mathrm{vw})$ & - \\
\hline \multirow{8}{*}{$\begin{array}{l}\text { Overlapping } v_{3}\left(\mathrm{UO}_{2}\right)^{2+} \\
v_{3}\left(\mathrm{AsO}_{4}\right)^{3-}, v_{1}\left(\mathrm{AsO}_{4}\right)^{3-} \\
\text { and } v_{1}\left(\mathrm{UO}_{2}\right)^{2+}\end{array}$} & $930(\mathrm{sh})$ & - \\
\hline & $908(\mathrm{sh})$ & $908(\mathrm{sh})$ \\
\hline & - & $896(\mathrm{~ms})$ \\
\hline & - & $883(\mathrm{sh})$ \\
\hline & $830(\mathrm{vs})$ & $830(\mathrm{sh})$ \\
\hline & $819(\mathrm{sh})$ & $816(\mathrm{~s})$ \\
\hline & - & $801(\mathrm{sh})$ \\
\hline & - & $796(\mathrm{sh})$ \\
\hline $\mathrm{H}_{2} \mathrm{O}$ libration modes & $735(\mathrm{sh})$ & - \\
\hline \multirow{2}{*}{$v_{4}(\delta)\left(\mathrm{PO}_{4}\right)^{3-}$} & $668(w)$ & - \\
\hline & $615(\mathrm{vw})$ & - \\
\hline \multirow{2}{*}{$v_{4}(\delta)\left(\mathrm{AsO}_{4}\right)^{3-}$} & - & 449 (mw-broad) \\
\hline & - & $320(\mathrm{w}-$ broad $)$ \\
\hline $\begin{array}{l}v_{2}(\delta)\left(\mathrm{UO}_{2}\right)^{2+} \\
\text { possible coincidence } \\
\text { with } \mathrm{U}-\mathrm{O}_{\text {ligand }} \text { vibrations }\end{array}$ & - & $206(\mathrm{sh})$ \\
\hline Lattice modes & - & $191(\mathrm{~ms})$ \\
\hline
\end{tabular}

Raman spectra of metalodèvite were presented, which seem to be similar with the present assignment of metakirchheimerite spectra (Tab. 4).

\subsubsection{Stretching $\mathrm{O}-\mathrm{H}$, bending $\mathrm{H}-\mathrm{O} \ldots \mathrm{H}$ vibrations}

A sharp infrared band at $3521 \mathrm{~cm}^{-1}$ (Fig. 6) is assigned to the $v \mathrm{OH}$ stretching vibration of only weakly hydrogen bonded water molecules, whereas a broad infrared band at $3123 \mathrm{~cm}^{-1}$, also attributed to the $v \mathrm{OH}$ stretching vibrations, is connected with more strongly hydrogen bonded water molecules. According to Libowitzky (1999), the tops of these bands are close to the $\mathrm{O}-\mathrm{H}$... O hydrogen bonds 2.94 and $2.69 \AA$, respectively. This proves that structurally non-equivalent hydrogen-bonded water molecules are present in the crystal structure of metakirchheimerite. This interpretation is supported by the fact that three corresponding infrared bands are observed in the region of the $v_{2}(\delta) \mathrm{H}_{2} \mathrm{O}$ bending vibrations at 1617 (shoulder), 1568 (maximum) and $1526 \mathrm{~cm}^{-1}$ (Fig. 6), and also by thermogravimetric analysis (Vochten and Goeminne 1984). The crystal structure analysis of synthetic metakirchheimerite (Locock et al. 2004) proved the presence of three symmetrically distinct $\mathrm{H}_{2} \mathrm{O}$ molecules held in the crystal structure only by hydrogen bonding, whereas five other $\mathrm{H}_{2} \mathrm{O}$ molecules are coordinated with the interlayer 


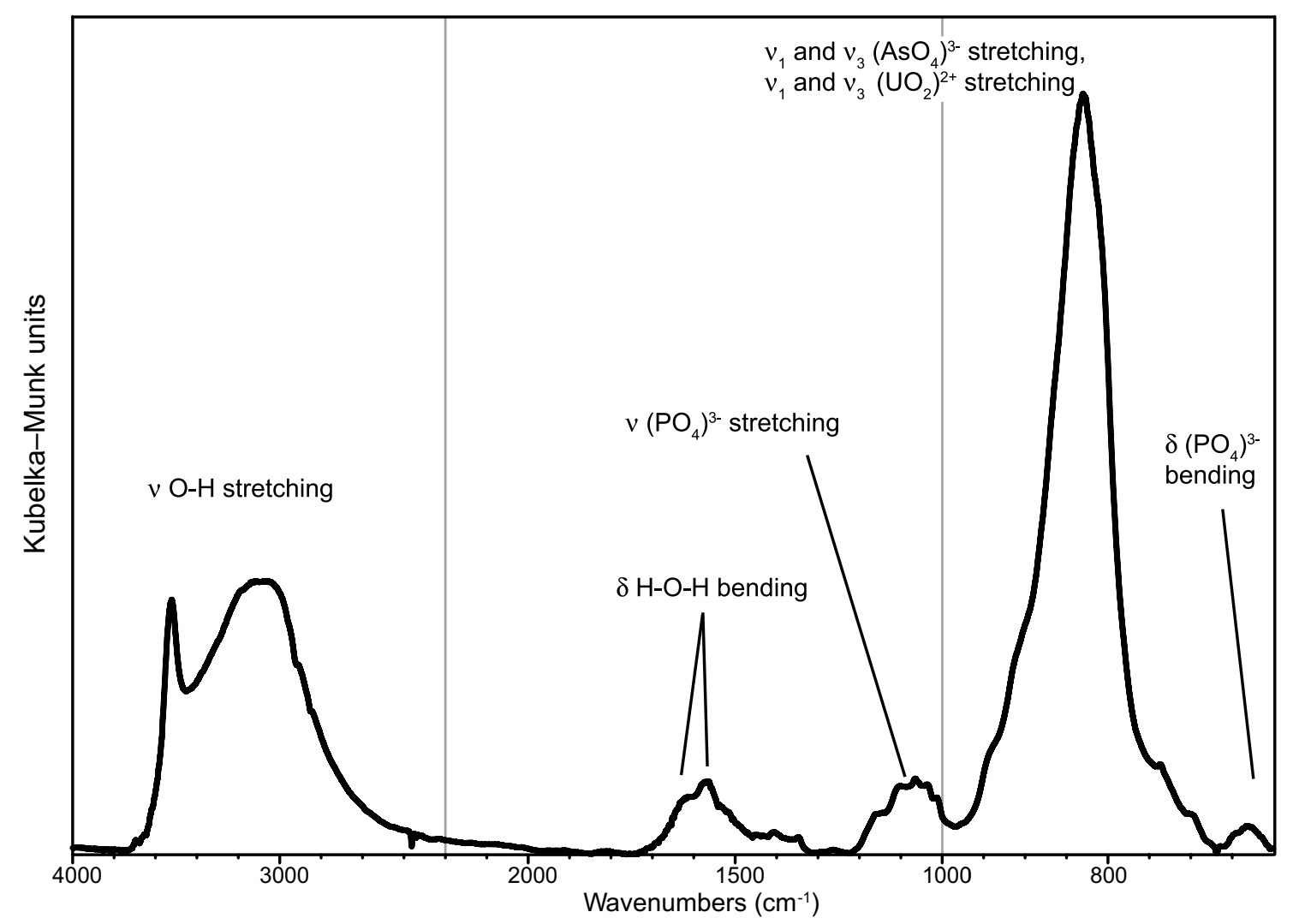

Fig. 6 The infrared spectrum of metakirchheimerite from Jáchymov. The assignments of the vibration bands are displayed for clarity.

$\mathrm{Co}^{2+}$ atom. A network of hydrogen bonding was proposed by Locock et al. (2004), based on $\mathrm{O} \cdots \mathrm{O}$ interatomic distances in the range 2.7 to $3.2 \AA$ (Fig. 1). A band at 735 $\mathrm{cm}^{-1}$ (a shoulder with maximum) was assigned to libration modes of water molecules (Čejka 1999).

\subsubsection{Vibrations of the uranyl $\left(\mathrm{UO}_{2}\right)^{2+}$ groups and $\left(\mathrm{AsO}_{4}\right)^{3--} /\left(\mathrm{PO}_{4}\right)^{3--}$ polyhedra}

The vibration modes assignment of these units is rather difficult due to extensive overlapping of the bands in the region between ca. 950-800 $\mathrm{cm}^{-1}$ (Figs 6-7), regarding stretching modes of $\mathrm{AsO}_{4}$ groups and uranyl ion. Based on empirical relation given by Bartlett and Cooney (1989) the following $\mathrm{U}-\mathrm{O}$ bond lengths were obtained from the wavelengths that could correspond to the uranyl stretching vibrations, observed in the both spectra: the infrared bands (Fig. 6) and/or shoulders at $930 \mathrm{~cm}^{-1}\left[v_{3} ; 1.763\right]$, $908 \mathrm{~cm}^{-1}\left[v_{3} ; 1.779\right], 830 \mathrm{~cm}^{-1}\left[v_{1} ; 1.781\right]$ and $811 \mathrm{~cm}^{-1}$ $\left[v_{1} ; 1.800\right]$; the Raman bands (Fig. 7) and/or shoulders at $908 \mathrm{~cm}^{-1}\left[v_{3} ; 1.779\right], 896 \mathrm{~cm}^{-1}\left[v_{3} ; 1.788\right], 883 \mathrm{~cm}^{-1}\left[v_{3}\right.$; 1.797], $816 \mathrm{~cm}^{-1}\left[v_{1} ; 1.795\right]$ and $801 \mathrm{~cm}^{-1}\left[v_{1} ; 1.810\right]$. But all these vibrations may be assigned to $\left(\mathrm{AsO}_{4}\right)^{3-}$ stretching modes, too. Chernorukov et al. (2000) observed in the infrared spectrum of a synthetic analogue of metakirch- heimerite in this region following bands at $952 \mathrm{~cm}^{-1}\left[\mathrm{v}_{3}\right.$; 1.749] $\left(v_{3} \mathrm{UO}_{2}{ }^{2+}\right), 896 \mathrm{~cm}^{-1}\left[v_{3} ; 1.786\right]$ and $812 \mathrm{~cm}^{-1}\left[v_{1}\right.$; 1.799] [ $\mathrm{AsO}_{4}{ }^{3-}$ ]; in the brackets is cited the assignment proposed by Chernorukov et al. (2000). Based on singlecrystal study of synthetic metakirchheimerite analogue, Locock et al. (2004) reported average $\mathrm{U}-\mathrm{O}$ bond length (uranyl) of $1.781 \AA[\mathrm{U}(1)-\mathrm{O} 1.765(3)$ and 1.809(3) $\AA, \varnothing$ $1.787 \AA$; U(2)-O 1.774(3) and 1.777(3) $\AA, \varnothing ~ 1.755(5)$ $\AA$ ]. These values can be compared with those inferred from the Raman and infrared spectra. A comparison of experimental Raman and infrared spectra is displayed in Fig. 8, which can be helpful in understanding the overlapping bands and their assignments. As an approximation, it can be assumed that infrared shoulders at $930 \mathrm{~cm}^{-1}$ and $908 \mathrm{~cm}^{-1}$ are connected with the $v_{3} \mathrm{UO}_{2}^{2+}$ antisymmetric stretching vibration, the strong band at $830 \mathrm{~cm}^{-1}$ (IR) to the $v_{3} \mathrm{AsO}_{4}{ }^{3-}$ triply degenerate antisymmetric stretching vibration overlapping the $v_{1}\left(\mathrm{UO}_{2}\right)^{2+}$ and $v_{1}$ $\left(\mathrm{AsO}_{4}\right)^{3-}$ stretching bands, that must have lower intensities than antisymmetric modes in the infrared spectrum. The Raman shoulder at 908 and maximum at $896 \mathrm{~cm}^{-1}$ are assigned to the $v_{3}\left(\mathrm{UO}_{2}\right)^{2+}$ symmetric stretching vibration. The number of bands is caused by presence of two non-equivalent $U$ atoms in the crystal structure, which is supported by the results of factor group analysis. 


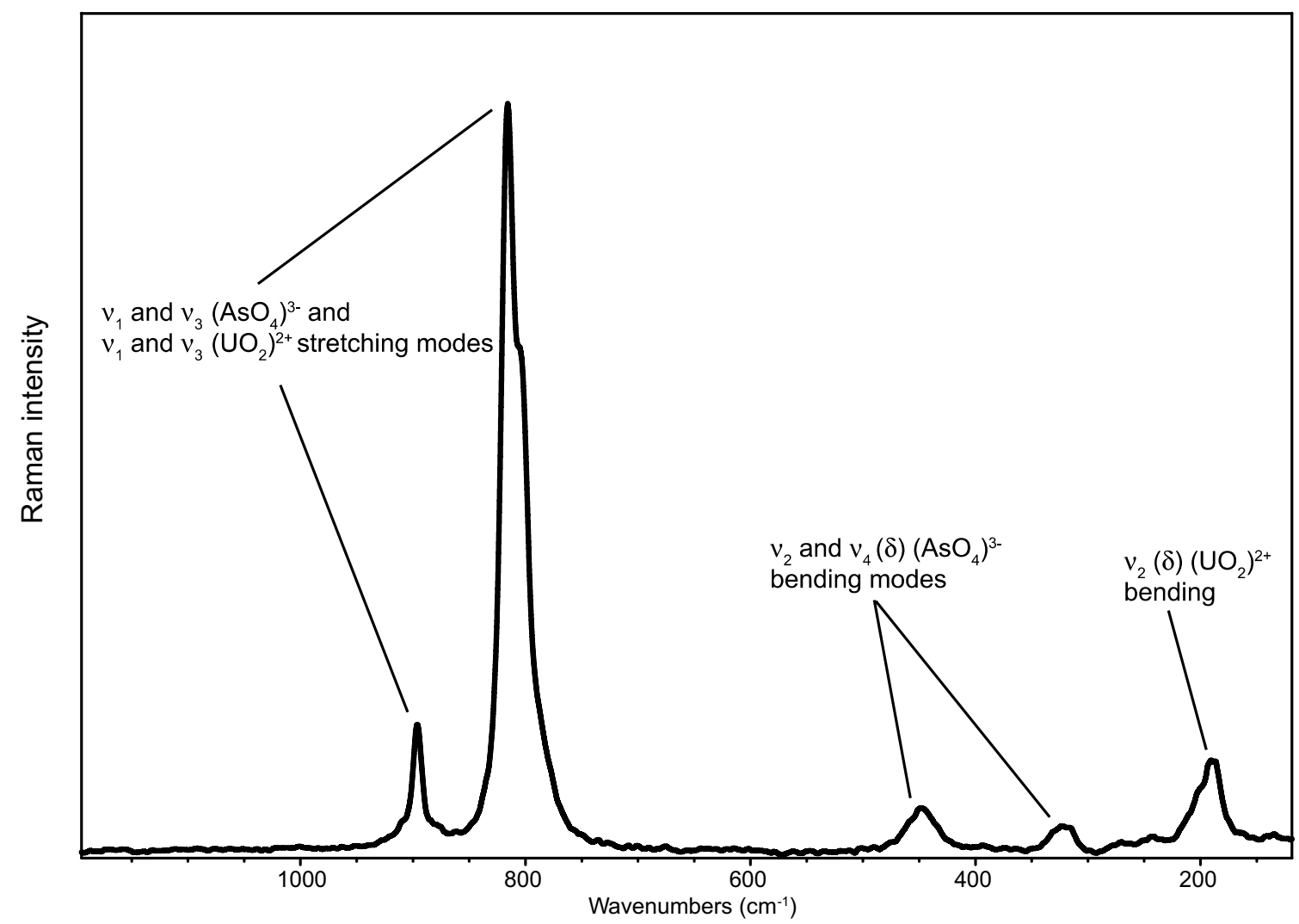

Fig. 7 Raman spectrum of metakirchheimerite from Jáchymov with tentatively assigned vibration bands.

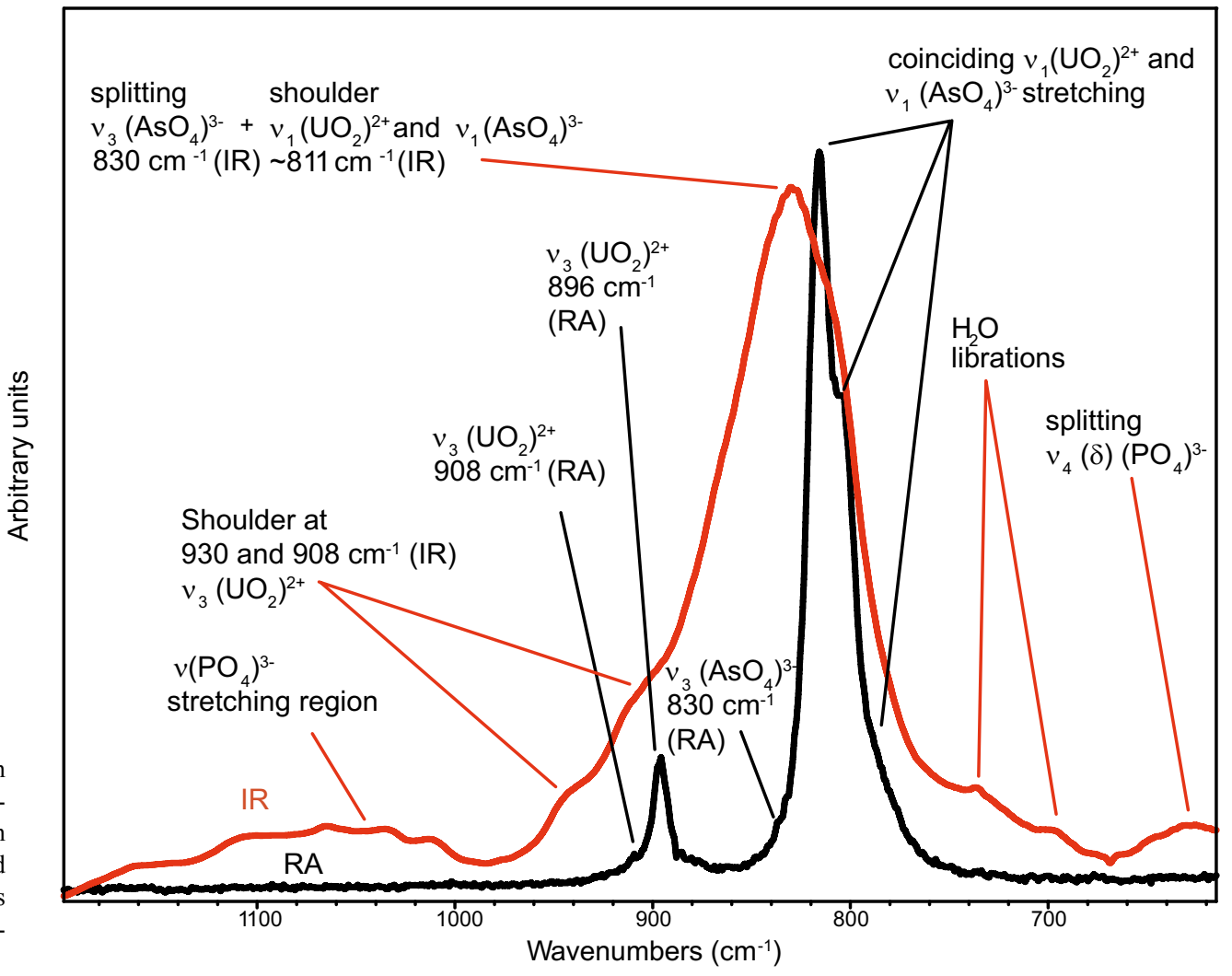

Fig. 8 A comparison of Raman and infrared spectra of metakirchheimerite in the region of the overlapping $\left(\mathrm{UO}_{2}\right)^{2+}$ and $\left(\mathrm{AsO}_{4}\right)^{3-}$ stretching vibrations with tentatively assigned vibration bands. 
The shoulder at $830 \mathrm{~cm}^{-1}$ (RA) is connected with the $v_{3}$ $\left(\mathrm{AsO}_{4}\right)^{3-}$ antisymmetric stretching vibration. The intensive band with maximum at $816 \mathrm{~cm}^{-1}$ and with shoulders at 801 and $790 \mathrm{~cm}^{-1}$ belongs to the overlapping $v_{1}\left(\mathrm{UO}_{2}\right)^{2+}$ and $v_{1}\left(\mathrm{AsO}_{4}\right)^{3-}$ symmetric stretching vibration modes.

Infrared weak bands at 1109,1065 and $1007 \mathrm{~cm}^{-1}$ (Fig. 6) are attributed to the split $\mathrm{v}_{3} \mathrm{PO}_{4}^{3-}$ triply degenerate antisymmetric stretching vibration, and those at 650 and $630 \mathrm{~cm}^{-1}$ to the splitting $v_{4}(\delta) \mathrm{PO}_{4}^{3-}$ triply degenerate bending vibration. Broad Raman bands at 449 and $320 \mathrm{~cm}^{-1}$ (Fig. 7) are assigned to the $v_{4}(\delta) \mathrm{AsO}_{4}{ }^{3-}$ triply degenerate and $\mathrm{v}_{2}(\delta) \mathrm{AsO}_{4}{ }^{3-}$ doubly degenerate bending vibrations, respectively. A shoulder at $206 \mathrm{~cm}^{-1}$ is attributed to the $v_{2}(\delta) \mathrm{UO}_{2}{ }^{2+}$ bending vibration and that at 191 $\mathrm{cm}^{-1}$ may be connected with lattice modes.

\section{Notice on the occurrence of metakirchheimerite in the nature}

There are only two reported localities of metakirchheimerite worldwide: Sophia Mine in Wittichen, Germany (Walenta 1958) and Jáchymov ore district, Czech Republic (Ondruš et al. 2003c). The reasons of metakirchheimerite rarity are only poorly understood at present. The association of cobalt arsenides with uranium minerals is relatively common in uranium deposits not only in the Czech Republic (e.g., Zálesí near Javorník, Jáchymov ore district) but also e.g. in the Schlema deposit (Germany) or at Bou Azzer (Morocco). Commonly, within these localities, uraninite occurs on the calcite-dolomite veins. During the alteration of primary mineralization, the local environment is probably highly saturated in $\mathrm{Mg}$ from dolomite, which leads to the formation of metanováčekite instead of Ni-Co uranyl arsenates. The observations on the natural samples from Jáchymov indicate that alteration of cobalt arsenides dominantly results in crystallization of erythrite, even in the environment rich in $\left(\mathrm{UO}_{2}\right)^{2+}$ ions. In the Jáchymov ore district, a Ni-dominated hydrated uranyl arsenate - metarauchite (IMA 2008-050) (Plášil et al. 2008) is by far more abundant than metakirchheimerite. The chemical similarity of $\mathrm{Co}$ and $\mathrm{Ni}$ suggests that an analogous behaviour of these elements could be expected in supergene processes. It indicates that under oxidation conditions cobalt and nickel exhibit slightly different behaviour but the problem is actually poorly understood (V. Ettler, person. comm. 2009). On the other hand, the more or less inconspicuous appearance of metakirchheimerite, in contrast to secondary minerals coloured by $\mathrm{Co}^{2+}\left(\right.$ or $\mathrm{Ni}^{2+}$ ), may be an important factor. It is possible that metakirchheimerite remains unnoticed at numerous localities.
Acknowledgements The kind help of Ivan Němec (Charles University, Prague) and Vladimír Machovič (Institute of Chemical Technology, Prague) with spectroscopic measurements as well as the assistance of Radek Škoda (Masaryk University, Brno) with electron microanalyses are highly acknowledged. The valuable comments by Andrew Locock (University of Alberta, Canada) are highly acknowledged, as well as his kind favour in providing us with the schematic picture of the metakirchheimerite crystal structure and reprint permission. Our thanks are due to both anonymous reviewers for their valuable comments as well as big chief Vojtěch Janoušek and handling editor Milan Novák. The authors are grateful for the financial support by the Grant Agency of the Charles University in Prague (GAUK no. 17008/2008), the Ministry of Culture of the Czech Republic (DE07P04OMG004) and the Ministry of Education of the Czech Republic (MSM0021620855).

Electronic supplementary material. The GPS coordinates of the studied samples are available online at the Journal web site (http://dx.doi.org/10.3190/jgeosci.057).

\section{References}

Bartlett J R, Cooney R P (1989) On the determination of uranium-oxygen bond lengths in dioxouranium(VI) compounds by Raman spectroscopy. J Mol Struct 193: 295-300

Catalano JG, Heald SM, Zachara JM, Brown Ge, JR (2004) Spectroscopic and diffraction study of uranium speciation in contaminated vadose zone sediments from the Hanford Site, Washington State. Environ Sci Tech 38: $2822-2828$

Catalano JG, McKinley JP, Zachara JM, Heald SM, Smith SC, BRown GE, JR (2006) Changes in uranium speciation through a depth sequence of contaminated Hanford sediments. Environ Sci Tech 40: 2517-2524

ČEJKA J (1999) Infrared spectra and thermal analysis of the uranyl minerals. In: BuRns PC, EwING RC (eds) Uranium: Mineralogy, Geochemistry and the Environment. Rev Mineral 38: 521-622

Chernorukov N G, Suleimanov E V, Dzhabarova S T, BARCH S V (2000) Synthesis and investigation of compounds of the series $\mathrm{A}^{\mathrm{II}}\left(\mathrm{B}^{\mathrm{V}} \mathrm{UO}_{6}\right)_{2} \cdot \mathrm{nH}_{2} \mathrm{O}\left(\mathrm{A}^{\mathrm{II}}-\mathrm{Mn}, \mathrm{Fe}\right.$, $\mathrm{Co}, \mathrm{Ni}, \mathrm{Cu}, \mathrm{Zn} ; \mathrm{B}^{\mathrm{V}}-\mathrm{P}, \mathrm{As}$ ). Radiokhim 42: 16-31 (in Russian)

Coelho A A, Cheary R W (1997) X-ray Line Profile Fitting Program, XFIT. Program manual, pp 1-4

Fayek M, Burns PC, Guo YX, Ewing RC (2000) Microstructures associated with uraninite alteration. J Nucl Mater 277: 204-210 
Finch RJ, EwING RC (1992) The corrosion of uraninite under oxidizing conditions. J Nucl Mater 190: 133-156

Finch RJ, Murakami T (1999) Systematics and paragenesis of uranium minerals. In: BuRNs PC, EwING RC (eds) Uranium: Mineralogy, Geochemistry and the Environment. Rev Mineral 38: 521-622

Finch RJ, Buck E, Finn P, Bates J (1999) Oxidative corrosion of spent $\mathrm{UO}_{2}$ fuel in vapour and dripping groundwater at $90{ }^{\circ} \mathrm{C}$. In: Scientific Basis for Nuclear Waste Management XXII, Materials Research Symposium Proceedings 556: 432-438

Finn P, Hoh J, Wolf S, Slater S, Bates J (1996) The release of uranium, plutonium, cesium, strontium, technetium and iodine from spent fuel under unsaturated conditions. Radiochim A74: 65-71

KIRCHHEIMER FW (1963) Uran und Seine Geschichte. E Schweizerbartsche Verlagsbuchhandlung, Stuttgart, pp 1-372

Laugier J, Bochu B (2002) Celref, version 3. Domaine Universitaire, Grenoble, France

LiBowitzky E (1999) Correlation of O-H stretching frequencies and $\mathrm{O}-\mathrm{H} . . . \mathrm{O}$ hydrogen bond lengths in minerals. Monat Chem 130: 1047-1059

Locock AJ (2007a) Crystal chemistry of actinide phosphates and arsenates. In: KRIVOvichev SV, BuRNS PC, Tananaev IG (eds) Structural Chemistry of Inorganic Actinide Compounds, Chapter 6. Elsevier, Amsterdam, pp 217-278

Lососк AJ (2007b) Trends in actinide compounds with the autunite sheet-anion topology. Proc Russian Mineral Soc 136: $115-137$

Locock AJ, Burns PC, Flynn TM (2004) Divalent transition metals and magnesium in structures that contain the autunite type sheet. Canad Mineral 42: 1699-1718

Murakami T, Ohnuki T, Isobe H, Sato T (1997) Mobility of uranium during weathering. Amer Miner 82: 888-899

Nabar M A, Iyer VJ (1977) Preparation and crystallographic characterization of uranyl double arsenates $\mathrm{M}^{\mathrm{II}}\left(\mathrm{UO}_{2} \mathrm{AsO}_{4}\right)_{2} \cdot 8 \mathrm{H}_{2} \mathrm{O}(\mathrm{M}=\mathrm{Zn}, \mathrm{Ni}$ and $\mathrm{Co})$. Bull Soc franç Minéral Cristallogr 100: 272-274

Ondruš P, Veselovský F, Gabašová A, Hloušek J, Šrein V (2003a) Geology and hydrothermal vein system of the Jáchymov (Joachimsthal) ore district. J Czech Geol Soc 48: 3-18

Ondruš P, Veselovský F, Gabašová A, Hloušek J, Šrein V, Vavř́ín I, Skála R, SejKora J, Drábek M (2003b) Primary minerals of the Jáchymov ore district. J Czech Geol Soc 48: 19-147

Ondruš P, Veselovský F, Gabašová A, Hloušek J, Šrein $\mathrm{V}$ (2003c) Supplement to secondary and rock-forming minerals of the Jáchymov ore district. J Czech Geol Soc 48: $149-155$

Ondruš P, Veselovskí $F$, Gabašová A, Drábek M, Dobeš P, Malý K, Hloušek J, Sejkora J (2003d) Ore-forming processes and mineral parageneses of the Jáchymov ore district. J Czech Geol Soc 48: 157-192

Pléšil J (2009) Crystal chemistry of selected natural hydrated uranyl arsenates and phosphates of divalent transition metals and magnesium. Unpublished MSci. thesis, Faculty of Science, Charles University, Prague, pp 1-113

Plášil J, Sejkora J, Novák M, ŠKácha P, Čejka J, Ondruš P, Veselovský F, Laufek F (2008) Metarauchite, $\mathrm{Ni}\left(\mathrm{UO}_{2}\right)_{2}\left(\mathrm{AsO}_{4}\right)_{2} \cdot 8 \mathrm{H}_{2} \mathrm{O}$, confidential information of the new mineral approval, IMA, pp 1-6

Pouchou JL, PICHOIR F (1985) "PAP” ( $\varphi \rho Z)$ procedure for improved quantitative microanalysis. In: ARMSTRONG JT (ed) Microbeam Analysis. San Francisco Press, San Francisco, pp 104-106

Procházka R (2007) Natural corrosion of the uraniumcolored historical glasses. J Non-Cryst Sol 353 (18-21): 2052-2056

Procházka R, Ettler V., Goliáš V, Klementová M, Mihaljevič M, Šebek O, Strnad L (2009) A comparison of natural and experimental long-term corrosion of uranium-colored glass. J Non-Cryst Sol 355: 2134-2142

Shueneman RA, Khaskelis A I, Eastwood D, van Ooij W J, BurgrafF F W (2003) Uranium oxide weathering: spectroscopy and kinetics. J Nucl Mat 323: 8-17

Vochten R, Goeminne A (1984) Synthesis, crystallographic data, solubility and electrokinetic properties of meta-zeunerite, meta-kirchheimerite and nickel-uranylarsenates. Phys Chem Minerals 11: 95-100

WALENTA K (1958) Die sekundären Uranmineralien des Schwarzwaldes. J geol Landesamt Baden-Würtemberg 3: $17-51$

WALENTA K (1964) Beiträge zur Kenntnis seltener Arsenatmineralien unter besonderer Berücksichtigung von Vorkommen des Schwarzwaldes. Tscherm Mineral Petrog Mitt 9: 111-174

Wronkiewicz D, Bates J, Gerding T, Veleckis E, Tani B (1992) Uranium release and secondary phase formation during unsaturated testing of $\mathrm{UO}_{2}$ at $90^{\circ} \mathrm{C}$. J Nucl Mater 190: $107-127$

Wronkiewicz D J, Bates JK, Wolf SF, Buck EC (1996) Ten-year results from unsaturated drip tests with $\mathrm{UO}_{2}$ at $90{ }^{\circ} \mathrm{C}$ : implications for the corrosion of spent nuclear fuel. J Nucl Mater 238: 78-95 


\section{Nová data pro metakirchheimerit z Jáchymova (St. Joachimsthal), Česká republika}

Metakirchheimerit byl nalezen pouze na několika málo vzorcích pocházejících ze žíly Jan Evangelista na Štolovém patře šachty Svornost v Jáchymově. Metakirchheimerit na vzorcích tvoří krystalické agregáty sestávající z tenkých tabulkovitých krystalů do velikosti $0.1 \mathrm{~mm}$. Krystaly jsou velmi křehké, světle růžové až oranžové barvy a silného skelného lesku. Krystalické agregáty metakirchheimeritu narůstají na karbonátovou žilovinu s vtroušenými primárními minerály - arsenopyritem, uraninitem a skutteruditem. Studovaný metakirchheimerit je produktem sub/recentního zvětrávání primárních minerálních fází vzniklým v prostředí opuštěného důlního díla. Na základě elektronové mikroanalýzy lze chemické složení studovaného metakirchheimeritu vyjádřit empirickým vzorcem $\left(\mathrm{Co}_{0.53} \mathrm{Mg}_{0.25} \mathrm{Ni}_{0.08} \mathrm{Zn}_{0.07}\right.$ $\left.\left.\mathrm{Fe}_{0.05} \mathrm{Ca}_{0.03}\right)_{\Sigma 1.01}\left(\mathrm{UO}_{2}\right)_{2.07}\left[\left(\mathrm{AsO}_{4}\right)_{1.99}\left(\mathrm{PO}_{4}\right)_{0.01}\right)\right]_{\Sigma 2.00} .8 \mathrm{H}_{2} \mathrm{O}$ (na bázi As $+\mathrm{P}=2$ apfu). Metakirchheimerit z Jáchymova vykazuje značný podíl izomorfní metanováčekitové komponenty. Rentgenový práškový difrakční záznam metakirchheimeritu odpovídá triklinické symetrii zjištěné studiem syntetického analogu na monokrystalovém difraktometru. Vypřesněné mř́žkové parametry pro triklinickou prostorovou grupu $P-1$ jsou $a 7.210(4) \AA, b 9.771(6) \AA, c 13.252(9)$ $\AA, \alpha 75.39(4)^{\circ}, \beta 83.94(6)^{\circ}, \gamma 81.88(6)^{\circ}$ s objemem $V$ 892(1) $\AA^{3}$. V infračerveném i Ramanově spektru metakirchheimeritu se projevují valenční a deformační vibrace $\left(\mathrm{UO}_{2}\right)^{2+},\left(\mathrm{AsO}_{4}\right)^{3-},\left(\mathrm{PO}_{4}\right)^{3-}$ a molekulární $\mathrm{H}_{2} \mathrm{O}$. Vibrační projevy jednotlivých molekulárních skupin byly přiřazeny jednotlivým vlnočtům. Vazebné délky U-O v uranylu délky vodíkových vazeb, které byly odvozeny studiem vibračních spekter, jsou konsistentní se strukturními daty uváděným pro syntetický analog metakirchheimeritu. 